\title{
i
}

\section{La prigione e la libertà: due testimonianze del dialogo di Fortini con i classici \\ Pietro Cataldi}

ABSTRACT: Analisi di due volumetti di scritri critici di Franco Fortini: Dialoghi col Tasso (1999) e Le rose dell'abisso (1991, cinque conversazioni radiofoniche su Tasso, Dante, Leopardi, Manzoni e Pascoli).

PAROLE CHIAVE: Fortini critico, Dialoghi col Tasso; Le rose dell'abisso; critica e poesia.

"La mia prigione vede più della tua libertà». Questo verso con cui Fortini chiude Diario linguistico, una poesia dedicata a Pasolini, può fungere da chiave di lettura del suo interesse appassionato per Tasso, di cui offre compiuta testimonianza il volumetto Dialoghi col Tasso (a cura di Donatello Santarone, Bollati Boringhieri, Torino 1999). "Ringrazio / il Signore che mi ha voluto restringere» recita d'altra parte il Monologo del Tasso a Sant'Anna compreso nel fortiniano Paesaggio con serpente, e posto ad apertura della silloge critica, con intelligente funzione di epigrafe. Il nesso prigionia/libertà, ovvero il bisogno costitutivo di essere ristretti per poter vedere più larghi spazi, è una delle costanti ermeneutiche della riflessione di Fortini su Tasso e al tempo stesso uno dei posizionamenti stranianti adottati da Fortini poeta per sancire, in uno, la colpa e la forza della letteratura. In questo senso, Tasso gli appare una esemplare figura della condizione di ostaggio reclamata anche per se stesso, ovvero una prefigurazione del modo violento e languido con cui la contraddizione dell'essere poeta si ricompone e armonizza nella forma. La forma tassesca ha la forza di dire con lo stesso vigore tanto la lacerazione quanto l'unità, tanto cioè l'affronto 
storico patito dal soggetto quanto il privilegio di conoscerlo ed esorcizzarlo. Perché la scrittura di Tasso (nella Gerusalemme soprattutto) è capace di caricarsi delle due energie contemporaneamente, mettendo in moto quel contrasto ben noto tra forza centrifuga e forza centripeta, tra fascinazione del proibito e sua condanna senza appello, tra scorrevolezza musicale e intoppo singhiozzante, tra lirica (secondo un luogo comune della critica) e narrazione epica.

Questa possibilità di leggere il Tasso di Fortini come chiave di accesso alla poetica e all'ideologia del critico e del poeta moderno è seducente e fertile. Anche perché non c'è forse nessun altro scritro fortiniano, neppure teorico o autobiografico, in cui sia dato di cogliere con più chiarezza alcuni nodi segreti della sua personalità, e i nessi tra aspetti e forme disparati di essa. Così che questo libretto ha, fra gli altri, il merito, per quanti vorranno farci i conti, di non disgiungere, in Fortini, il poeta dall'ideologo e dal critico, come accade ormai con frequenza, se non si vuole "sentenziare a morte» l'uno e l'altro.

E tuttavia, gli scritti su Tasso qui raccolti meritano di essere considerati innanzitutto per il loro valore specificamente critico, indipendentemente dal rimando alla personalità del loro autore.

C'è da ricavare innanzitutto una lezione di metodo, che la ottima "Premessa" di Mengaldo ben mette in luce: «il merito grande di Fortini studioso dello stile e delle forme tassiani non sta tanto nel porre in relazione di reciprocità o omologia fatti di quell'ordine con fatti extrastilistici ed extraformali; ma, ben di più, nel cercare di stabilire l'isomorfia dei vari piani del discorson (p. 13). Esemplari sono, da questo punto di vista, le considerazioni sulla figura del chiasmo e sulla tecnica dell'enjambement. Il chiasmo risulta una delle categorie costruttive dello stile tassesco (tra i molti esempi addotti riporto qui solo "A me l'onor, la morte a me si deve»: II, 29, 5). In esso, che rompe l'ordine del parallelismo ma risarcisce la rottura con una nuova forma di simmetria, si fondono infatti l'istanza centrifuga e quella centripeta, l'intento di ordine (e di ortodossia anche architettonica) e la pulsione verso lo smembramento; così da essere "un manto sonoro su angoscia latente». «Ebbene questa figura retorica diventa un emblema stilistico anche di elementi più 
grandi come gruppi di versi all'interno di singole ottave fra loro o fra personaggi» (p. 114). Quanto all'enjambement, decisivo nella versificazione tassesca, Fortini vi vede la «sottolineatura di una norma nel momento in cui la si contesta. In questo senso" diviene una tecnica usimbolica di tutto l'atteggiamento poetico del Tasso, che esige la legge nell'atto medesimo in cui la negan (p. 62). L'analisi accurata dei vari impieghi stilistici ed espressivi dell'enjambement, condotta con una finezza da esperto "metricologo", rivela inoltre come la "pausa irrazionale» (Fubini) di fine verso concorra ora alla "contrazione martellata e dura, centripeta", ora a "larghi gesti di eloquenza o furore patetico e grandioso", cioè ai udue estremi, l'uno di compressione e di ravvolgimento, l'altro di espansione e di scatenamenton (p. 63).

La lezione di metodo critico è insomma quella che si ricava anche dagli studi critici fortiniani più noti (e raccolti nei Saggi italiani e nei Nuovi saggi italiani); cioè la capacità di risalire dal particolare stilistico e formale a un sistema di poetica e all'impianto psicologico, fino ai nessi con l'ideologia dell'autore e con quella del suo tempo, se non addirittura alle coordinate culturali e storico-sociali circostanti. I passaggi sono a volte meditati e dialettici, a volte bruciati nel cortocircuito. La tensione di Lukács verso la totalità si incrocia, nei casi migliori, con l'ossessione benjaminiana del particolare; e il metodo critico di Fortini risulta così, fra l'altro, a sua volta un frutto di lacerazione fra istanze anche diverse e contrapposte, nonché del potente tentativo di conferire a esse unità. Ma la lezione riguarda in ogni caso l'esigenza di non ridurre la critica letteraria a descrizione della superficie linguisticoformale, pure indagata con la massima cura e con competenza; né di dare luogo a una pretestuosa interrogazione del testo in veste filosofica, benché poi Fortini non rinunci mai a interpellare il possibile contenuto di verità dell'oggetto studiato. Ciò che conta è insomma la saldatura fra il momento descrittivo e quello interpretativo e attualizzante, cioè fra filologia e filosofia; avvenga ciò attraverso una serie di puntuali mediazioni, avvenga invece con la messa a confronto bruciante di referto strettamente stilistico e di diagnosi storico-filosofica. Al cospetto di un orizzonte critico che troppo spesso si riduce o a solo filologismo descrittivo, senza capacità o interesse a interrogare la verità (storica e filosofica) del testo, o a gratuito esercizio 
parafilosofico, senza rispetto della datità del proprio oggetto di studio, la lezione metodologica di questi scritti fortiniani mi pare di notevole rilievo (e di scontrosa inattualità).

Così come attuali risultano le cinque conversazioni radiofoniche raccolte in un altro volume, anch'esso curato da Donatello Santarone, interlocutore prezioso di Fortini: Le rose dell'abisso. Dialoghi sui classici italiani (Bollati Boringhieri, Torino 2000). Tra le cinque conversazioni, svoltesi nel giugno del 1991, Fortini affronta di nuovo Tasso, e poi Dante, Leopardi, Manzoni e Pascoli. Quale che sia il terreno del confronto (ma sempre autori del canone maggiore), Fortini si mostra interessato a due ordini di tensione (o di contraddizione) interna: tra particolare e insieme, ovvero qualche volta anche fra "dono" della forma e durezza del referto ideologico, e tra necessaria datità storico-filologica e attualità possibile e utile. Soprattutto al cospetto di Leopardi e di Manzoni, due autori amati e frequentati assiduamente da Fortini, si legge in queste pagine la virtù quasi scomparsa di tenere insieme serietà della ricerca e interesse per il presente entro cui la ricerca si colloca. Nel capitolo leopardiano, per esempio, la questione del rapporto tra pensiero e sua formalizzazione implica la volontà di prendere posizione tanto contro la dominante sopravvalutazione della felicità irrazionale dell'arte quanto contro la rimozione, in campo materialistico, della sua specificità difficilmente razionalizzabile. Nel momento in cui colloca la propria posizione all'interno di un dibattito critico pertinente e aggiornato (dialogando con Maria Corti se discorre di Dante, con Zatti se è la volta di Tasso, e con Timpanaro o Binni se si parla di Leopardi), Fortini non si limita mai a un confronto limitato all'oggetto; ma vede comunque il significato latamente politico e attuale delle posizioni altrui e della propria. Lo specialismo non è la zona franca di un sereno dialogo tra eguali e privilegiati, ma il privilegiato territorio di un conflitto generale da cui nessun margine può dirsi escluso. Neppure quel territorio al nostro sguardo così enigmatico nel quale si manifesta la formalizzazione artistica. Fortini è anzi sempre attratto dal momento in cui l'ideologia e la psiche diventano forma, o che si tratti di Leopardi che con la seconda sepolcrale compie una «ricognizione della salma di Aspasia» (secondo una accattivante intuizione: p. 
74), o che Manzoni sia colto in flagrante ambivalenza tra la volontà di denunciare l'orrore del potere, anche religiosamente assistito, e il bisogno di censurarne le punte più crude e irredimibili. Ė frugando tra le pieghe di una contraddizione siffatta che viene opportunamente valorizzato l'abbinamento editoriale, preteso caparbiamente da Manzoni, ma ignorato di solito dagli editori moderni, tra Promessi sposi e Storia della colonna infame: "Quando è finito il romanzo con quello strano capitolo finale nel quale tutto sembra messo a posto [...], quando il lieto fine persino melenso sembra assicurato, Manzoni fa un improvviso ritorno indietro, ci riporta nel cuore della storia, nel cuore della peste, nel cuore dell'orrore [...]» (p. 103).

Linteresse di Fortini per le procedure della formalizzazione si volge sempre infine a questo "cuore dell'orrore»; e il manierismo è perciò la sua dimora, anche quale poeta in proprio. Anche in questo caso, tuttavia, sarà bene non disgiungere l'utilità di queste pagine per la comprensione più fonda della poetica di Fortini poeta dalla utilità per la migliore conoscenza degli autori trattati; sarà bene, cioè, non negare al lavoro critico di Fortini la legittimità di un atto conoscitivo. Certo, non sarà un caso che l'interesse critico fortiniano si appunti sempre infine su autori presso $i$ quali la crisi delle strutture ideologiche e dei sistemi conoscitivi assuma la forma di una crisi delle strutture e dei sistemi di linguaggio, e il rinnovamento della lingua divenga un'esigenza tanto forte quanto, in genere, contraddittoria e impedita. $\mathrm{Da}$ questo punto di vista, l'interesse di Fortini può egualmente appuntarsi sulla eroica scommessa di senso di Dante, per il trascinamento utopico e figurale che la sua stessa edificazione linguistica porta con sé; su Tasso, per la tensione che nella poesia di questo viene spalancata tra espressione piena dell'io e sua negazione, cioè tra lingua del desiderio e lingua della repressione (psicologica e storica); su Leopardi, per la necessità sua di fare $i$ conti con la classicità quale rovina; su Manzoni, diviso tra funzionalizzazione sociale della letteratura e coscienza della sua inutilizzabilità storica; su Pascoli, infine, quale convivenza di recupero cimiteriale dell' antico e di apertura necessaria al nuovo, cioè porta (non unica ma fondamentale) del Novecento.

Il lettore che ne abbia voglia, e soprattutto il giovane lettore che senta il coraggio bastante, potrà ben partire da questi due volumetti di agile mole e di scorrevole 
lettura per poi misurare le posizioni fortiniane nei loro momenti più canonici $\mathrm{e}$ controllati. Se può essere formulato un augurio a concludere questa breve ricognizione, sia allora che questo lettore esista, che esista questo giovane lettore: che esista ancora.

RESUMO: Análise de dois pequenos volumes de escritos criticos de Franco Fortini: Dialoghi col Tasso (1999) $e$ Le rose dell'abisso (1991, cinco conversas radiofonicas sobre Tasso, Dante, Leopardi, Manzoni e Pascoli).

PALAVRAS-CHAVE: Fortini critico; Dialoghi col Tasso; Le rose dell'abisso; critica e poesia. 